\title{
Evaluating Short-Term Spatio-Temporal Tropospheric Variability in Multi-Temporal SAR Interferograms Using LES Models
}

\author{
Fengming Hu, Member, IEEE, Kevin C. Helfer, A. Pier Siebesma, and Ramon F. Hanssen, Senior Member, IEEE
}

\begin{abstract}
Atmospheric delay has a significant impact on synthetic aperture radar (SAR) interferometry, inducing spatial phase errors and decorrelation in extreme weather condition. For Low Earth Orbit (LEO) SAR missions, the atmosphere can be considered as being spatio-temporally frozen due to the short integration time. Geosynchronous (GEO) SAR missions, however, have short revisit times and extensive imaging coverage but with a longer integration time. As a result, GEOSAR interferograms can provide continuous deformation monitoring and integrated refractivity for weather forecasting. However, as the troposphere may vary significantly within the integration time, this may lead to a degradation during focusing and decorrelation of the InSAR pair. Here we simulate a time-series refractivity distribution with a high spatio-temporal resolution, for a fair-weather situation using an advanced Large Eddy Simulation (LES) model, to show the spatio-temporal variability of the troposphere on short time scales. Given GEO orbit parameters with different viewing angles along both azimuth and range directions, corresponding time-series of tropospheric interferograms are obtained based on the SAR geometry, and the impacts of different parameters are compared. Tropospheric delay is found to vary rapidly and a lead to phase gradient exceeding one cycle within a few minutes. Yet, for periods of less than $\sim 15$ minutes, a frozen-flow approximation may be successful to mitigate atmospheric decorrelation. Consequently, GEOSAR imaging should be iterative to compensate the atmospheric effects.
\end{abstract}

Index Terms-tropospheric delay, multi-temporal InSAR, large-eddy simulation

\section{INTRODUCTION}

$\mathbf{S}$ YNTHETIC aperture radar (SAR) plays an important role in remote sensing, by observing the earth's surface with a large coverage and acquiring information about its physical properties, which is successfully used in many applications such as land cover classification and monitoring flooding area [1]. Using two consecutive SAR acquisitions, the interferometric phase denotes the path delay between two observations along the line of sight, reflecting the topographic height difference, surface motion and atmospheric effects [2].

This research was funded with the Hydroterra Phase-0 Science and Requirement project by ESA.

F. Hu is with the Key Laboratory for Information Science of Electromagnetic Waves (MoE), Fudan University, Handan Road 220, Shanghai, 200433, China, and also with the Department of Geoscience and Remote Sensing, Delft University of Technology, 2628 Delft, The Netherlands (email: fm_hu@fudan.edu.cn).

K.C. Helfer, A.P. Siebesma, and R.F. Hanssen are with the Department of Geoscience and Remote Sensing, Delft University of Technology, Delft, 2628 $\mathrm{CN}$, the Netherlands (email: k.c.helfer, a.p.siebesma, r.f.hanssen@tudelft.nl).
Thus, it is possible to monitor the topography, deformation by earthquake, volcanic and land subsidence $[3,4,5]$.

Although the atmospheric delay is regarded as noise in conventional interferometric (In)SAR, many studies have shown the capability of Interferometric Synthetic Aperture Radar (InSAR) for providing maps of integrated refractivity with high precision and spatial resolution. The spatial variability of the integrated refractivity is mostly sensitive to the distribution of water vapor [6, 7]. Currently, this product cannot be used for weather forecasting because of its poor temporal sampling relative to atmospheric timescales but it is possible to assimilate this product into the numerical weather prediction model for identifying extreme rain fall $[8,9]$.

Compared with Low Earth Orbit (LEO) SAR, geosynchronous (GEO) SAR has a short revisit time and large coverage of potentially more than $1000 \mathrm{~km} \mathrm{[10],} \mathrm{leading} \mathrm{to} \mathrm{an}$ opportunity to obtain real-time surface observation for fast disaster warning [11]. In addition, interferograms with extremely short temporal baselines enable the frequent and continuous production of integrated refractivity maps with high resolution. Near real-time integrated refractivity can improve weather forecasting and risk alarming of potential natural disaster since heavy rain can increase the risk of landslide [12]

When radio waves pass through the atmosphere, the velocity becomes slightly lower because the refractive index is higher than in vacuum, leading to spatially variable delays [13]. Errors induced by the atmosphere will affect the coherence of SAR signals and imaging quality. In addition, effects of the troposphere are more significant than that of the ionosphere for high-frequency system [14].

The troposphere delay can be divided into a hydrostatic, a wet, and a liquid term. The hydrostatic term depends on pressure and surface temperature, assuming to be a smooth signal, which can be predicted accurately using the Saastamoinen model [15]. In areas with significant topography, the hydrostatic term shows a topography-related phase variation, which can be estimated using a linear [16] or powerlaw model [17]. The wet part has significantly more spatiotemporal variability, which reduces the accuracy of estimating elevation and deformation from the interferometric phase. The effect of the wet delay is larger than that of the hydrostatic delay in SAR interferograms, and is more difficult to remove without external data. Different studies have been conducted to estimate the wet delay in interferograms, using GNSS [18] or weather models [19]. Liquid delay caused by clouds can be 
neglected since it only contributes an error of a few millimeters to the total delay [2].

Many studies analyzed the effects of troposphere on both quality of interferogram and radar imaging. The impact of the troposphere on radar imaging is considered using a spatiotemporal frozen model, also known as Taylor's hypothesis [20], in LEOSAR due to its short integration time and small image coverage. However, the integration time of a geosynchronous SAR (GEOSAR) can range from a few minutes to hours depending on the orbit parameters [21]. This means that the effects of the troposphere on imaging can't be ignored. [22] pointed out that the influence of both ionosphere and troposphere should be considered during the design of the GEOSAR system. [23] show that path errors caused by both ionosphere and troposphere will affect the position of the point targets, leading to range offsets and azimuth defocusing. These influences will increase in images with higher resolution and larger incidence angles [24]. [25] proposed a power spectrum model for modeling the turbulence random errors, showing the influence of the time-varying troposphere on radar imaging. [26] analyzed different types of temporal decorrelation and suggested that an advanced atmospheric model could provide wet delay prediction with a high precision based on a numerical model [27]. Since the illumination is longer for GEOSAR, the space-time variability of the atmosphere may be so significant that it can affect the image formation. The space and time decorrelation induced by atmospheric turbulence and their impact on SAR focusing are shown in [28, 29].

Thus far, the temporal variability of the refractivity distribution has not been investigated using a large eddy simulation (LES) model with high spatio-temporal resolution. In this paper, we introduce a standard approach to generate atmospheric interferograms, i.e., synthetic interferograms only sensitive to atmospheric delay variability, showing the time-varying characteristics of the troposphere. First, the Dutch Atmospheric Large Eddy Simulation (DALES) model and its output atmospheric parameters are briefly reviewed. Then details of the transformation from model parameters to refractivity are introduced. In addition, the slant delays are calculated with the given orbit parameters. Finally, time series of atmospheric interferograms are generated using two types of interferogram generation and the impacts of their spatio-temporal characteristics on GEOSAR are shown correspondingly.

\section{INTERFEROMETRIC DELAY}

\section{A. Definition of the Slant Delay}

The two-way slant delay difference $2 \delta_{p}$ for a location $p$ at acquisition $t_{1}$ expressed as phase delay $\left(\psi_{p}\right)$ is given by

$$
\psi_{p, t_{1}}=\frac{4 \pi}{\lambda} \delta_{p, t_{1}} .
$$

where $\lambda$ is the radar wavelength. During the GEOSAR focusing, both spatial and temporal variability of the atmosphere induces decorrelation noise [29]. In this study, we neglect the decorrelation noise of SAR imaging induced by the space variability, since the main aim is to evaluate the spatiotemporal variability of the troposphere on short time scale.
Thus, the interferograms denoted in the followings could be regarded as ideal synthetic interferograms.

The observed phase difference $\phi_{p}$ in the interferogram between acquisitions $t_{1}$ and $t_{2}$ is

$$
\phi_{p}=\frac{4 \pi}{\lambda}\left(\delta_{p, t_{2}}-\delta_{p, t_{1}}\right) .
$$

Given the antenna position $a$, the delay at the image pixel $p$ is obtained by integrating the path along the line of sight using the refractivity $N$, which is defined as $[30,2]$ :

$$
\delta_{p, t}=10^{-6} \int_{p}^{a} N(x, y, z, t) d z .
$$

Considering all types of atmospheric delay, the original expressions of the refractivity can be written as [31]:

$$
N=k_{1} \frac{P_{d}}{T}+\left(k_{2} \frac{e}{T}+k_{3} \frac{e}{T^{2}}\right)+k_{4} \frac{n_{e}}{f^{2}}+k_{5} W
$$

where $P_{d}$ is partial pressure of dry air, $e$ denotes partial pressure of water vapor, $T$ is temperature, $n_{e}$ is the electron density per cubic meter, $f$ is the radar frequency and $W$ is the liquid water content. The constants used in this paper are $k_{1}=77.6 \mathrm{KhPa}^{-1}, k_{2}=70.4 \mathrm{KhPa}^{-1}, k_{3}=3.739 \times$ $10^{5} \mathrm{~K}^{2} \mathrm{hPa}^{-1}, k_{4}=4.028 \times 10^{7} \mathrm{~m}^{-3}$, and $k_{5}=1.45 \mathrm{~m}^{3} \mathrm{~g}^{-1}$. The last two terms in Eq. (4) are the ionospheric term and the liquid term, which are not considered in this study. The other three terms are referred to as the tropospheric terms.

Assuming that the total atmospheric pressure is $P=P_{d}+e$, the tropospheric refractivity $N_{\text {tro }}$ is the summation of a hydrostatic and a 'wet' component:

$$
N_{\text {tro }}=k_{1} \frac{P}{T}+\left(k_{2}^{\prime} \frac{e}{T}+k_{3} \frac{e}{T^{2}}\right),
$$

where $k_{2}^{\prime}=k_{2}-k_{1} R_{d} R_{v}^{-1}$, with $R_{d}=287.053 \mathrm{~J} \mathrm{~K}^{-1} \mathrm{~kg}^{-1}$ and $R_{v}=461.524 \mathrm{~J} \mathrm{~K}^{-1} \mathrm{~kg}^{-1}$.

\section{B. Parameter Transformation}

The numerical atmospheric model used in this research is DALES, the Dutch Atmospheric Large Eddy Simulation model [32], which can provide reliable results for a multitude of atmospheric conditions. The LES uses $\theta_{L}$ and $q_{v}$ as prognostic variables and a diagnostic hydrostatic total pressure $p$ and liquid water specific humidity $q_{l}$. Since the tropospheric refractivity depends on partial pressure of water vapor, total pressure and temperature, see eq. (5), we need to convert the DALES parameters for $e$ and $T$. The definition of the specific humidity is

$$
q_{v}=\frac{m_{v}}{m_{t}}
$$

where $m_{v}$ denotes the specific mass of water vapour and $m_{t}=$ $m_{d}+m_{v}$ denotes the sum of the dry air specific mass and the specific mass of water vapour. Using the gas law and Dalton's law we can rewrite $q_{v}$ in terms of the vapor pressure as

$$
q_{v}=\epsilon \frac{e}{p+e(\epsilon-1)}
$$

where $\epsilon=R_{d} / R_{v}$. Inverting this gives the desired relation

$$
e=\frac{p}{\epsilon} \frac{q_{v}}{1+(1 / \epsilon-1) q_{v}} \simeq \frac{p}{\epsilon} q_{v}
$$




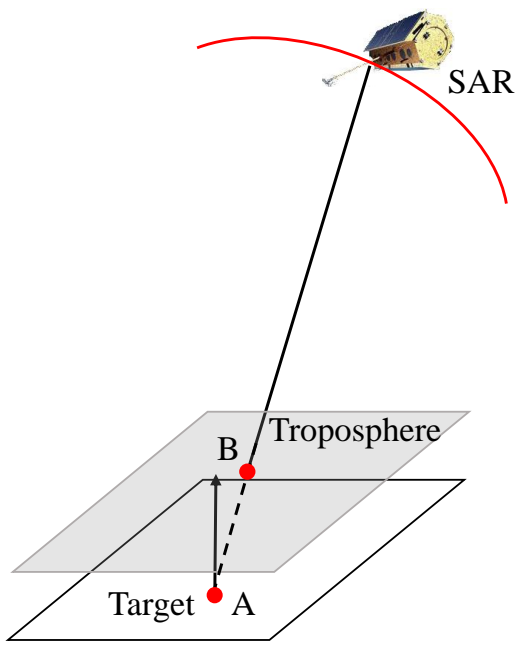

Fig. 1: Simplified sketch of radar signal propagation in the troposphere.

To compute the temperature $T$, we use the linearized form of the liquid water potential temperature $\theta_{L}$ in DALES $[33,30]$ :

$$
\theta_{L} \equiv \frac{1}{\pi} T-\frac{L_{v}}{c_{p} \pi} q_{l}
$$

where the latent heat of vaporization $L_{v}=2.25 \times 10^{6} \mathrm{~J} \cdot \mathrm{kg}^{-1}$, and the specific heat of dry air $c_{p}=1004 \mathrm{~J} \cdot \mathrm{K}^{-1} \cdot \mathrm{kg}^{-1}$. Inverting this allows to write the temperature in terms of $\theta_{l}$, $q_{l}$, and pressure $p$

$$
T=\pi \theta_{l}+\frac{L_{v}}{c_{p}} q_{l}
$$

where we use the Exner function defined as $\pi \equiv\left(\frac{p}{p_{0}}\right)^{R_{d} / c_{p}}$ and the standard pressure $p_{0}=1000 \mathrm{hPa}$.

Finally, with Eqs. (8) and (10), the 4-dimensional refractivity distribution can be obtained using Eq. (5) with all derived parameters.

We compute the tropospheric delay along the line of sight using ray-tracing [34] based on the SAR geometry. In Fig. 1, the radar signal passes the troposphere from point $B$ to illuminate $\operatorname{target} A$. Although the path of the signal is not a straight line, the contribution of the refractivity change due to the bending error is negligible for typical SAR incidence angles $[35,2]$. Thus, the total tropospheric delay for one acquisition is expressed as, cf. Eq. (3)

$$
\delta_{\text {tro }}=10^{-6} \int_{h_{A}}^{h_{\text {tro }}} N_{\text {tro }} d h,
$$

where $h_{A}$ is the elevation of target $A$ and $h_{\text {tro }}$ is the total height of the troposphere. The tropospheric delay is calculated using Eq. (1).

\section{Interferogram Generation}

Supposing that there are $m$ SAR acquisitions, $m-1$ independent interferograms are generated and the combination of the interferograms can be one of the following two types.
TABLE I: Parameters of the first time-series refractivity distribution

\begin{tabular}{ll}
\hline Items & values \\
\hline data interval $(\mathrm{s})$ & 10 \\
data period $(\mathrm{min})$ & 15 \\
scale $(\mathrm{km} \times \mathrm{km})$ & $49.3 \times 49.3$ \\
horizontal resolution $(\mathrm{m} \times \mathrm{m})$ & $40 \times 40$ \\
maximal height $(\mathrm{m})$ & 4500 \\
vertical resolution $(\mathrm{m})$ & 40 \\
\hline
\end{tabular}

In the single master approach, only one SAR image is chosen as the master image, and all interferograms are obtained by

$$
\phi_{p}^{i}=\operatorname{wrap}\left\{\psi_{p}^{i+1}-\psi_{p}^{1}\right\}
$$

where $\operatorname{wrap}\{\ldots\}$ denotes the wrapping operator [2]. In the daisy chain approach, all interferograms are generated using two subsequent SAR acquisitions, as given by

$$
\phi_{p}^{i}=\operatorname{wrap}\left\{\psi_{p}^{i+1}-\psi_{p}^{i}\right\} .
$$

Time series interferograms only show the relative changes of the interferometric phase over time. Retrieving the undifferenced integrated refractivity distribution corresponding to each SAR acquisition can be accomplished by adding the constraint on the assumption that the expectation of all observations is zero [36]. Other approaches use external data, such as MODIS [37] and GNSS data [7] to fix the observation in one of the epoch, the absolute values of the other epochs can be obtained correspondingly.

\section{Simulation AND Discussion}

We conduct large-eddy simulations using DALES, version 4.2. The simulation set-up is based on radiosonde and ground observations on 21 June 1997 at the Southern Great Plains site, an extensive atmospheric measurement site in Oklahoma and Kansas, USA [38]. The simulation covers an area of $49.3 \times 49.3 \mathrm{~km}^{2}$ and is characterised by shallow cumulus convection with a cloud cover between 20 and 30 percent, typical of continental mid-latitudes. After 6 hours of simulation time (when sufficient atmospheric turbulence and clouds have developed), we produce 3D output at a temporal resolution of 10 seconds for 15 minutes of simulation time to yield the 4D refractivity distribution. The relevant parameters are shown in Tab. I.

The most spatio-temporally variable parameters in the simulated refractivity distribution are the specific humidity $q$ and the liquid water potential temperature $\theta_{L}$, see Section II-B. Fig. 2 shows the vertical profile of these parameters, averaged over the entire domain per elevation level. The average of the specific humidity, see Fig. 2a, decreases significantly at higher altitudes, especially in the range from $1000 \mathrm{~m}$ to $2500 \mathrm{~m}$. This implies that most of the wet delay occurs lower than $2500 \mathrm{~m}$. The liquid water potential temperature, see Fig. $2 \mathrm{~b}$, stays nearly constant until $1000 \mathrm{~m}$ and increases significantly above $1000 \mathrm{~m}$. Finally, the difference in the specific humidity between the first $\left(t_{0}\right)$ and the last epoch $\left(t_{0}+15 \mathrm{~min}\right)$ as a function of height is shown in Fig. 2c, indicating that the water vapor is increasing during the simulated period. The 


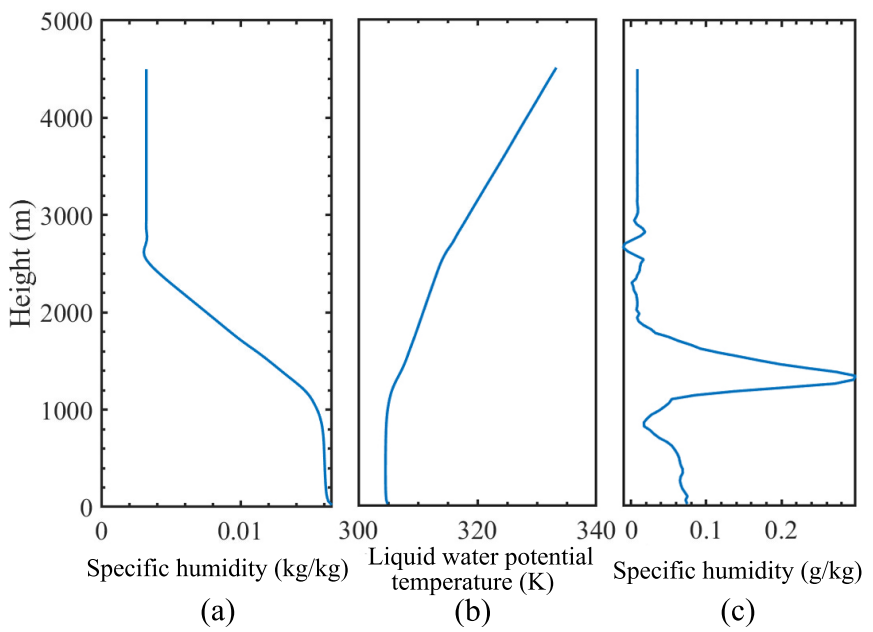

Fig. 2: Vertical distribution of (a) specific humidity, and (b) liquid water potential temperature, averaged over the entire domain at $t_{0}$. (c) Difference of the specific humidity between $t_{0}$, the first epoch, and $t_{0}+15 \mathrm{~min}$ (the last epoch) as a function of height.

maximum value of the difference is situated at a height of $1340 \mathrm{~m}$, which demonstrates that the total water vapor varies most significantly between 1000 and $2000 \mathrm{~m}$, which is the height where clouds are present.

In Eqs. (3) and (5), the tropospheric delay is divided into two parts, the hydrostatic and the wet delay. Fig. 3 shows the vertical delay for both components at $t_{0}$. for a subset of $6.7 \times 6.7 \mathrm{~km}$ of the original dataset.

While the absolute value of the hydrostatic delay is ${ }^{1}$ $\sim 914 \mathrm{~mm}$, which is larger than that of the wet delay, $\sim 218 \mathrm{~mm}$, the spatio-temporal variability range of the wet delay is more significant than that of both temperature and pressure, as follows from the spatial range of the hydrostatic delay $(\sim 1 \mathrm{~mm})$, which is much smaller than that of wet delay $(\sim 16 \mathrm{~mm})$, see Figs. $3 \mathrm{a}$ and $\mathrm{b}$.

The temporal variability of the hydrostatic and the wet delay, evaluated at one position, is shown in Figs. 4a and b, respectively. This shows that also the temporal variation of the hydrostatic delay is smaller than that of wet delay. The hydrostatic delay varies over $\sim 0.4 \mathrm{~mm}$, while the wet delay varies over $\sim 4 \mathrm{~mm}$ during the $15^{\prime}$ of the evaluation. Thus, the effects of the hydrostatic delay between subsequent acquisitions are extremely small, which may be negligible over the synthetic aperture integration of a geosynchronous InSAR mission.

To investigate the sensitivity of the observed signal delays to the atmospheric parameters, we compared $T^{-1}$ and $e$, cf. Eq. (5), with the delay, for a horizontal cross section of the delay at $t_{0}$. Figs. $5 \mathrm{a}$ and $\mathrm{b}$ show the spatial variation of tropospheric delay, here evaluated at a height of $900 \mathrm{~m}$, indicating that the spatial variation of the hydrostatic delay is highly correlated with the inverse temperature $(0.9996)$ while

${ }^{1}$ Note that the DALES model is evaluated up to a maximum elevation of $4.5 \mathrm{~km}$. As a consequence the total hydrostatic delay is less than typically experienced when dealing with the actual atmosphere in space geodetic methods.
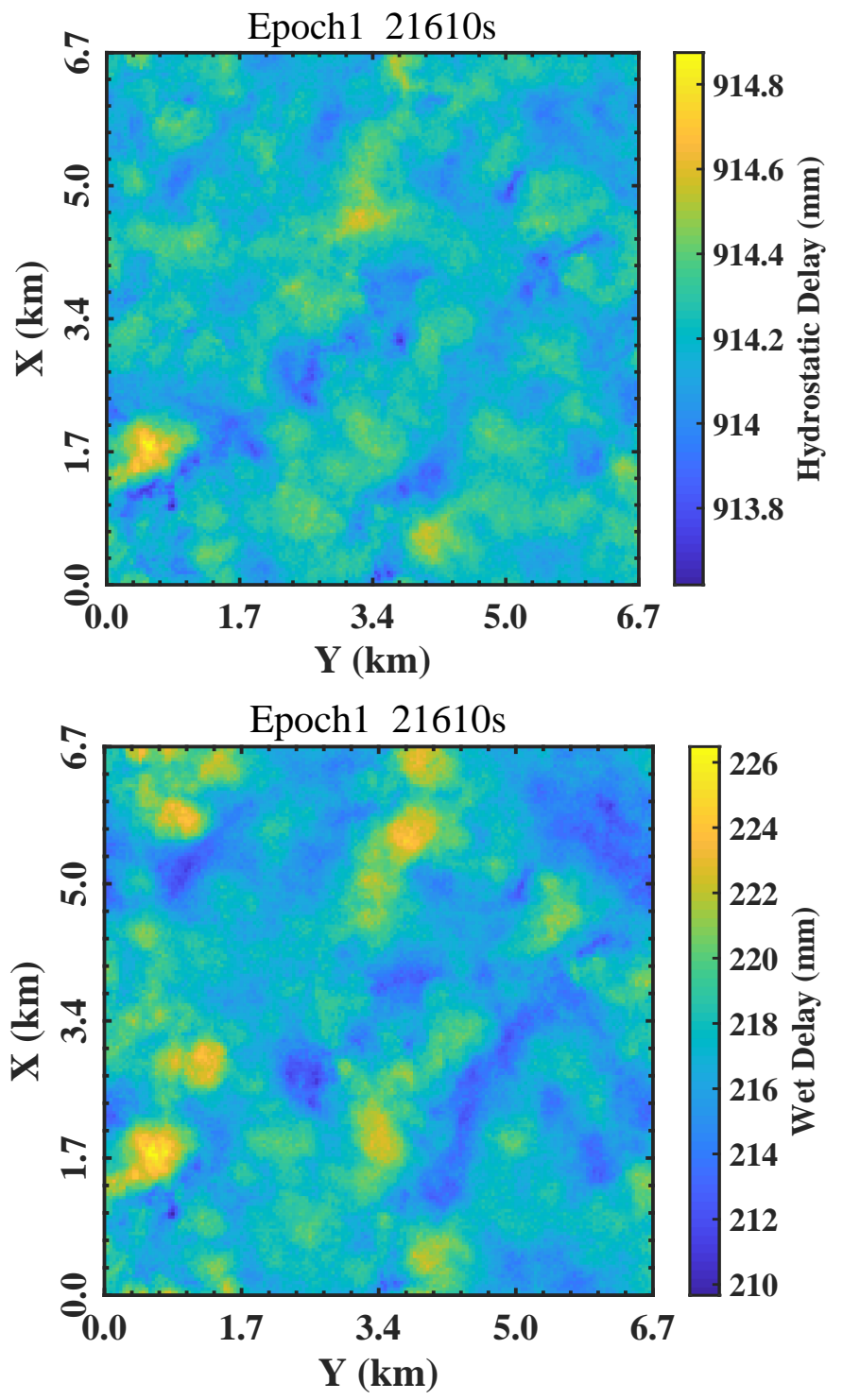

Fig. 3: Vertical tropospheric delay at $t_{0}$ for epoch 1: (a) hydrostatic delay, (b) wet delay. Note the difference in the colorbar values and ranges.

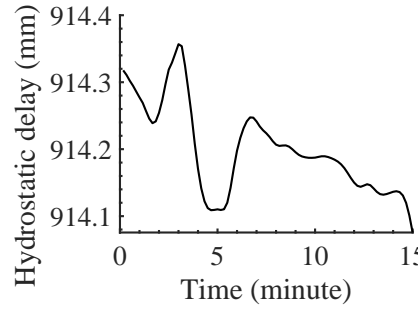

(a)

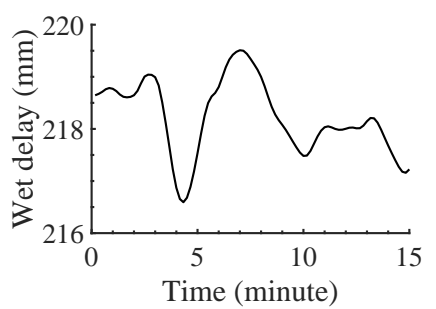

(b)
Fig. 4: Tropospheric delay time series for one location: (a) hydrostatic delay (b) wet delay. 


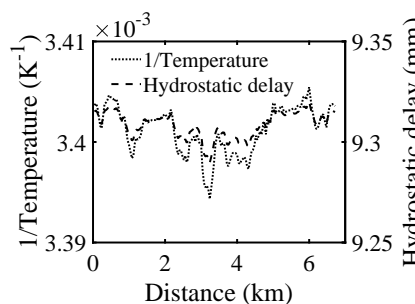

(a)

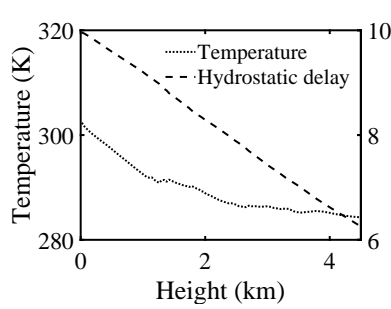

(c)

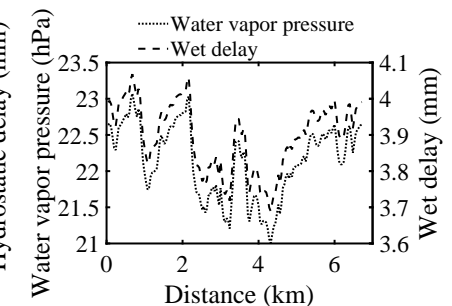

(b)

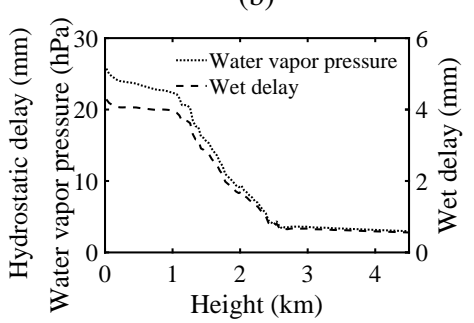

(d)
Fig. 5: Comparison of spatial changes between atmospheric parameters and tropospheric delay. (a) and (b) are horizontal variations at a height of $900 \mathrm{~m}$, and (c) and (d) are vertical variations. (a) and (c) are spatial variations of hydrostatic delay and temperature, (b) and (d) are spatial variations of water vapor pressure and wet delay.

that of the wet delay is very correlated with water vapor pressure (0.9998). Additionally, Figs. 5c and d show that the vertical variability of the tropospheric delay is much less than the horizontal variability. The vertical variability of hydrostatic delay is less correlated with the temperature while the vertical variability of the wet delay shows a strong correlation with water vapor pressure.

\section{A. Analysis of Vertical Delay Interferogram}

Using a C-band radar wavelength of $0.056 \mathrm{~m}$, two 'tropospheric' interferograms are derived, assuming vertical (zenith) delays, i.e., not projected to the line of sight. Fig. 6a shows a 15-minute (15') interferogram (epochs 1-90), and Fig. 6d a 10-second (10") interferogram (epochs 89-90), with a color bar range of $2 \pi$ rad and $1 \mathrm{rad}$, respectively, demonstrating the significant influence of a longer temporal separation. The short-term tropospheric interferogram signal can conveniently be disentangled into two temporal effects: (i) the 'frozen flow' drift of the entire refractivity distribution due to the prevailing wind [20], combined with (ii) turbulent advection during this time period. To investigate which of the two is dominant, we shift a copy of the tropospheric delay signal at $t_{0}$ to the position that maximizes the correlation with the observed interferogram between $t_{0}$ and $t_{i}$, yielding the frozen flow interferogram, see Fig. 6c. The difference between the observed interferogram and the frozen flow interferogram, i.e. Figs. 6a and c, could be regarded as the turbulence-only interferogram, see Fig. 6b. Comparison of Figs. $6 \mathrm{~b}$ and $\mathrm{c}$ shows a clear distinction between the frozen-flow and turbulenceonly interferograms. This is confirmed by their power spectra in Fig. 6e, which are used to analyze the energy distribution of the interferogram [2]. It shows a clear dominance of the frozen-flow component over the turbulence-only component for wavenumbers smaller than 0.7 cycles $/ \mathrm{km}$, hence scales larger than $1.4 \mathrm{~km}$. For smaller scales, there is no significant difference between the two components. The power spectra shown in Fig. 6e also provide a convenient stochastic expression of the variability of the atmospheric signal delay, indicating that the 'frozen-flow' approximation is a valid approximation within a time period of $15^{\prime}$. In other words, for time periods up to $15^{\prime}$, it is possible to simply consider the atmosphere as a fixed/frozen 3D refractivity distribution, that translates as a whole with the prevailing wind speed and direction.

Additionally, we compare the tropospheric power spectra between the unwrapped 15' interferogram and the corresponding single epoch images, see Fig. 7a. It shows that the atmospheric delay signals of one epoch and an epoch 15' later are similar in terms of their power and scaling behavior, and that the construction of an interferogram leads to a differential atmospheric signal that has more power over all of its spatial wavelengths. Fig. $7 \mathrm{~b}$ shows that the scaling behavior is a distinct function of the wavelengths, with the typical $-8 / 3$ scaling law for scales between 0.7 and $5 \mathrm{~km}$. The less steep power-law slopes for the other wavelengths indicate a more 'rough', or noise-like, signal for these domains. As the DALES model is evaluated up to a maximum elevation of $4.5 \mathrm{~km}$, the physical interpretation of the results should be limited to horizontal ranges less than $\sim 5 \mathrm{~km}$. Fig. $7 \mathrm{c}$ confirms that the power of the interferogram is about $\sqrt{2}$ times the power of the atmospheres in single epochs. We note that there is no way to confirm this in real-life situations, since singleepoch signals cannot be obtained unambiguously.

Fig. 8 demonstrates how the power in an interferogram, for different spatial scales, will increase with increasing temporal separation of the acquisitions. With increasing temporal separation, i.e., the color in Fig. 8a, we converge to a maximum. For example, at $200 \mathrm{~m}$ scales, the colors orange, yellow and green are obscured by the (longest) 15' temporal separation, while we do see lower power levels for temporal separations up to three minutes. This demonstrates a temporal ceiling of $\sim 3 \mathrm{~min}$ in the energy for scales around $200 \mathrm{~m}$, and of $\sim 10 \mathrm{~min}$ for scales around $10 \mathrm{~km}$. Again, we note that this understanding cannot be obtained from real-life interferograms, as such repeat frequencies are not available for study. Fig. $8 \mathrm{~b}$ demonstrates the amount of power we may encounter in making so-called 'daisy-chain' interferograms, which have a 10 second separation. These interferograms exhibit a similarly low absolute power, corresponding with very limited spatial variation between subsequent acquisitions, see Fig. 8 b.

Additionally, time series of the delay for five arbitrary locations are indicated by the colors in Fig 9. Figs. 9ac show the wrapped cumulative delays for $\mathrm{X}-, \mathrm{C}-$, and $\mathrm{L}-$ band, respectively. While the delays are identical due to the non-dispersive nature of the troposphere, the wrapping in the wavelength interval shows the significance of the signal variability per wavelength. Applying a daisy-chain approachcombining only subsequent SAR acquisitions-we find that the delay differences are very small, see Fig. 9d. This may be 
TABLE II: SAR orbit parameters

\begin{tabular}{ll}
\hline Items & values \\
\hline Semi-major axis $(\mathrm{km})$ & 42278.7 \\
Inclination (degree) & 51 \\
Argument of perigee (degree) & 165 \\
Eccentricity & 0 \\
wavelength (m) & 0.056 \\
incidence angle (degree) & 23 \\
\hline
\end{tabular}

negligible during conventional radar imaging.

\section{B. Analysis of Slant Delay Interferogram}

Given the satellite orbit parameters, slant delays and interferograms can be obtained using ray-tracing. Tab. II shows the main parameters of the SAR orbit we used in this simulation. Only the incidence angle should be adapted to consider the variety of GEOSAR and LEOSAR orbits. Due to the SAR geometry and location of the atmospheric model, the interferograms only cover a subset of the model's coverage. For example, in the case of $23^{\circ}$ incidence angle and $120^{\circ}$ heading angle, the coverage of the interferogram is $34 \times 36 \mathrm{~km}$.

Due to the side-looking geometry of SAR, tropospheric interferograms vary depending on both azimuth and range viewing angles. The main effects of the viewing angle are the size of the image coverage and the value of the total slant delay. The range of incidence angles increases with the increase of the coverage, for LEOSAR leading to a more significant linear change of the slant delay. As this ramp in the delay can be easily modeled, this effect can be accounted for.

To assess the influence of the temporally changing atmosphere, we show the total slant delay time series of the most and the least changing pixel in the domain, see Figs. 10a and $b$. During the 15 ' of the evaluation, the total slant delay varies over $\sim 40 \mathrm{~mm}$ for the most variable pixel (equivalent to 1.4 cycles for C-band), while it varies only within $\sim 3 \mathrm{~mm}$ for the least variable pixel. The latter is negligible during the radar imaging, while the former may have a significant effect in the SAR focusing.

A change in the viewing geometry during the SAR integration time results in a different path through the atmosphere, and will therefore also have an effect on the phase. This may influence the SAR focusing quality, which normally assumes a 'frozen' atmosphere during the aperture time [20]. We simulated extreme cases of a difference in viewing angles from $-23^{\circ}$ to $+23^{\circ}$, see the solid and dashed lines in Figs. 10a and $b$. Note that this difference is comparable for right-looking LEOSAR satellites with opposing headings (ascending and descending). For these extreme geometric differences, we see that the different path through the atmosphere may lead to more than $10 \mathrm{~mm}$ in delay difference over time, for the fair-

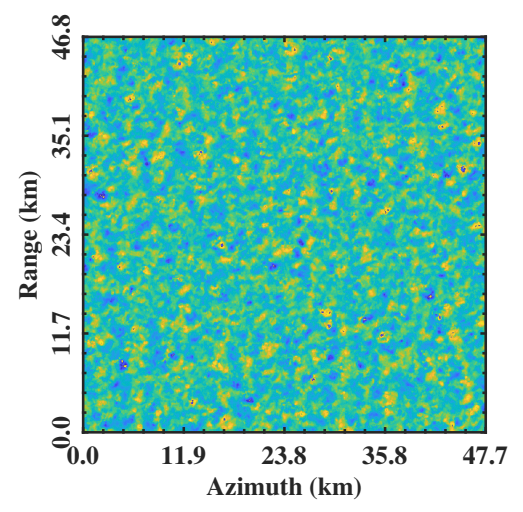

(a)

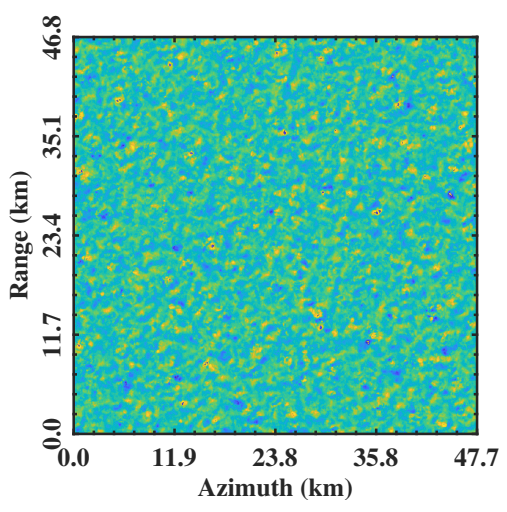

(b)

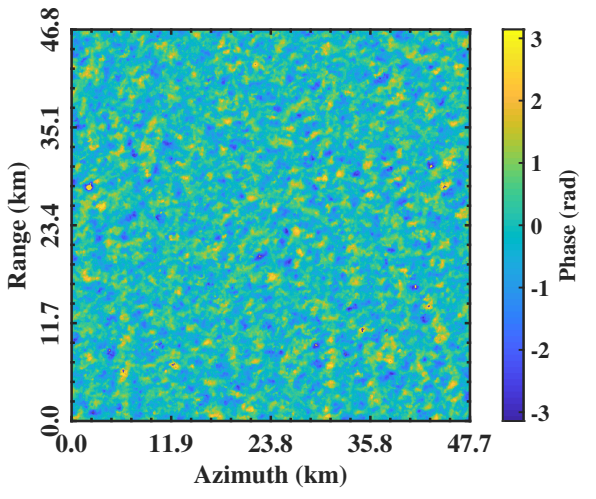

(c)

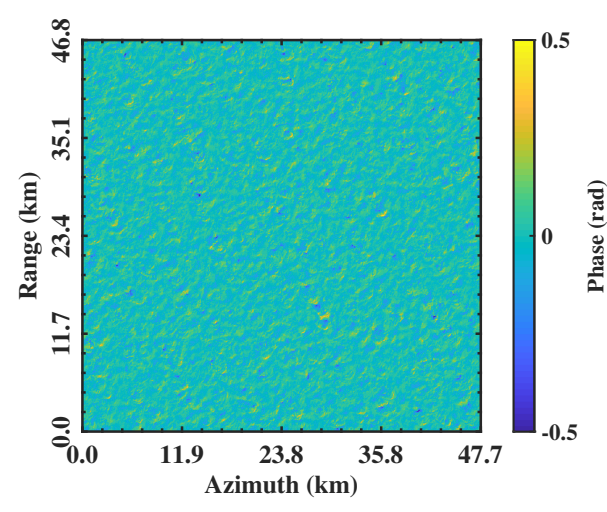

(d)

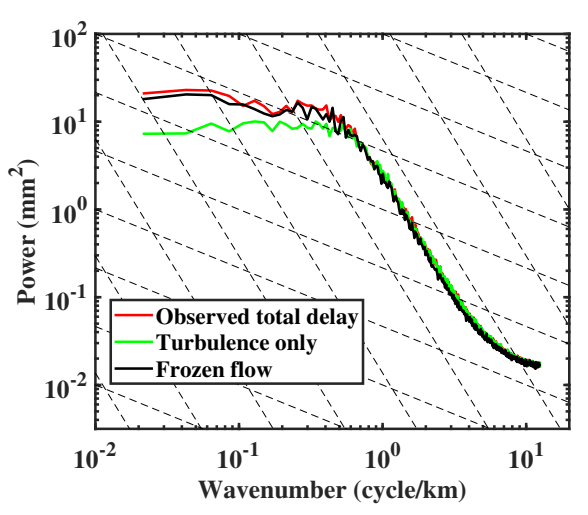

(e)

Fig. 6: Tropospheric interferogram over (a) 15 minute (epoch 1-90) and (d) 10 seconds (epoch 89-90). Note the difference in the colorbar range. (b) and (c) are turbulence-only and frozen flow interferograms of 15 minute interferograms, respectively. (e) shows the corresponding power spectra of (a), (b), and (c). 

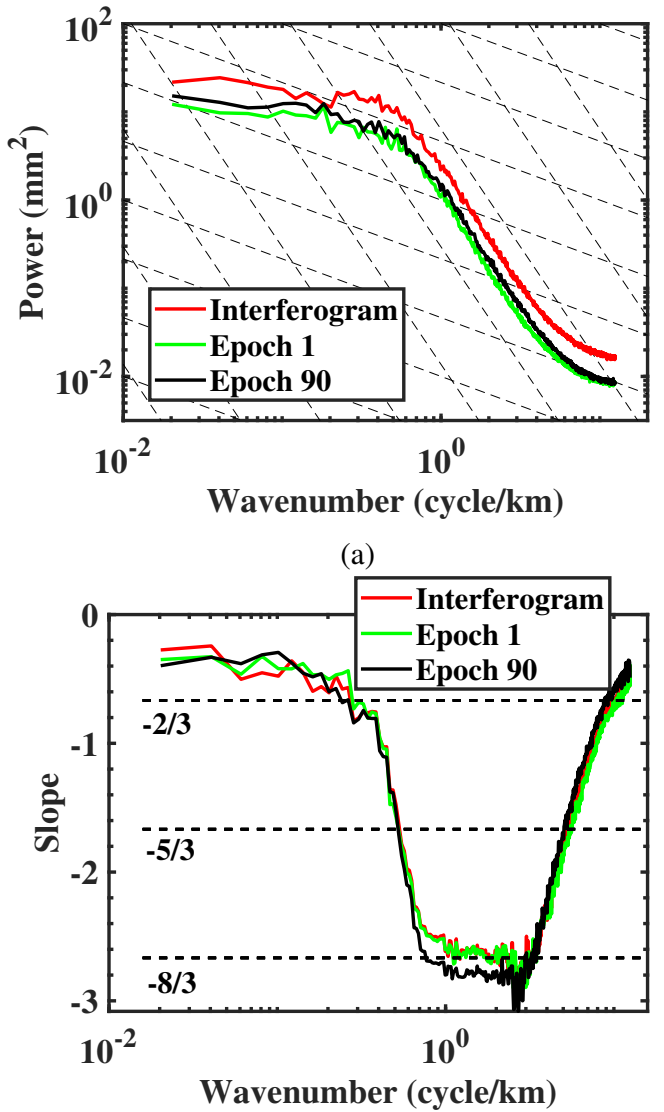

(b)

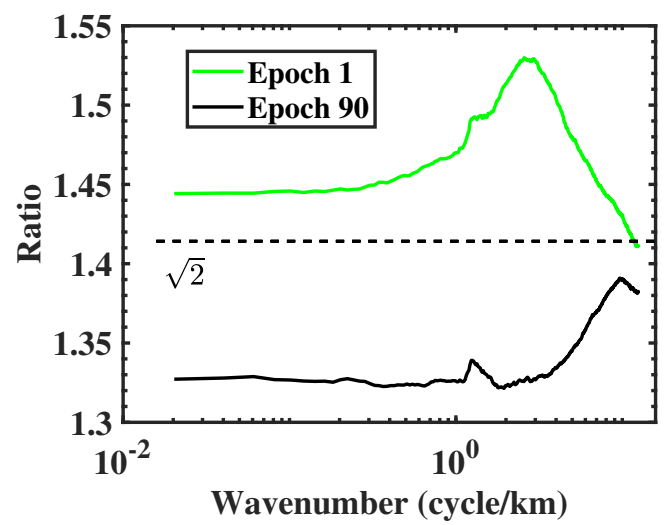

(c)

Fig. 7: Comparison of the power spectra between unwrapped 15 ' interferogram and the single epoch images. (a) Power spectra. (b) Slope of the power spectra, showing $-8 / 3$ and $-2 / 3$ scaling regimes for $0.7-5 \mathrm{~km}$ and $>8 \mathrm{~km}$ ranges, respectively. (c) Ratio of the power spectra between single epoch image and interferogram.

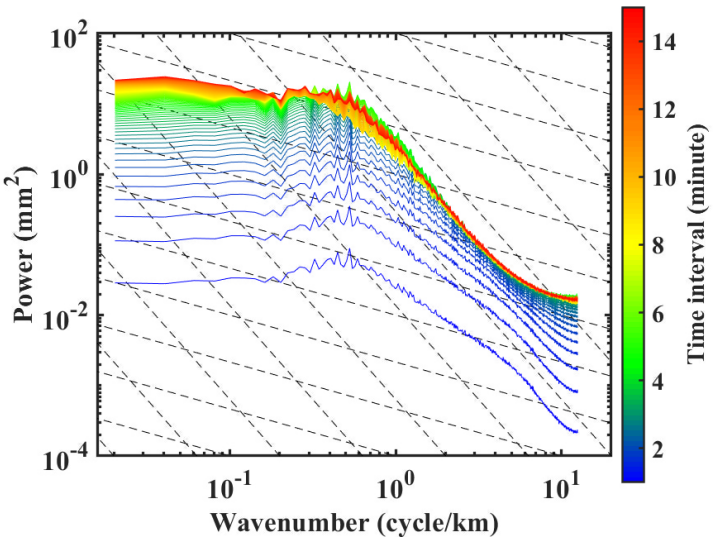

(a)

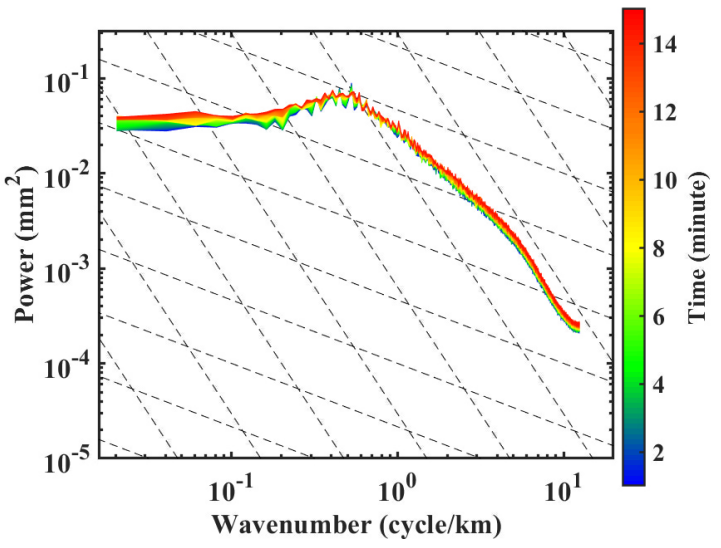

(b)

Fig. 8: One-dimensional power spectra interferogram combinations. (a) unwrapped single master interferograms, i.e., cumulatively increasing delays over time, (b) unwrapped daisy chain interferograms, i.e., only $10 \mathrm{sec}$ time differences. The dashed lines denote $-8 / 3$ and $-2 / 3$ power-law behavior.

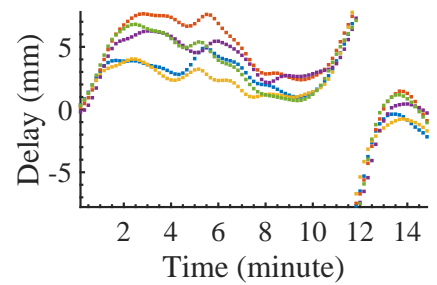

(a)

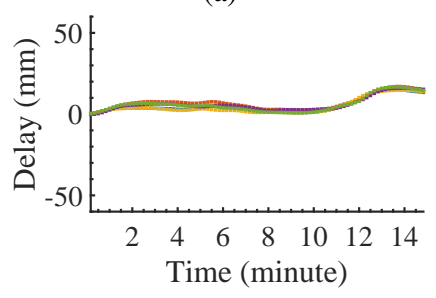

(c)



(b)

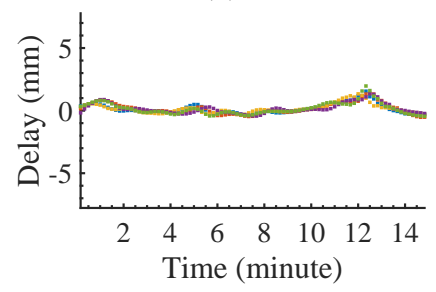

(d)
Fig. 9: Total wrapped delay time series of five arbitrarily selected pixels, expressed with colors, for different wavelengths: (a) single master in X-band, (b) single master in C-band, (c) single master in L-band, and (d) daisy chain in X-band. 


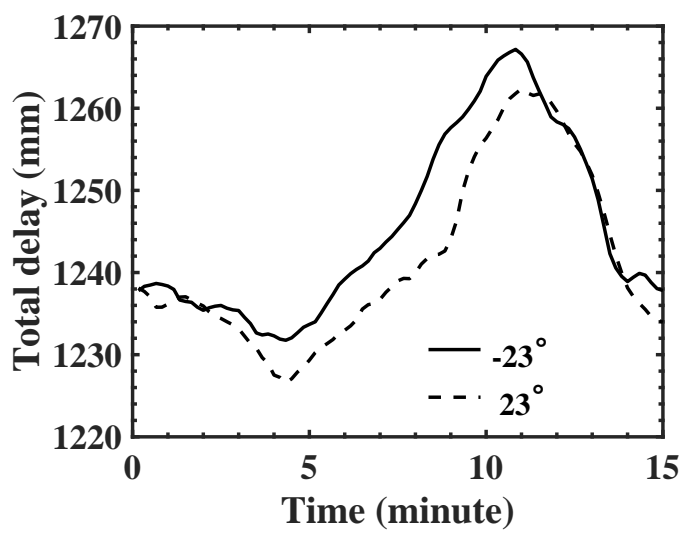

(a)

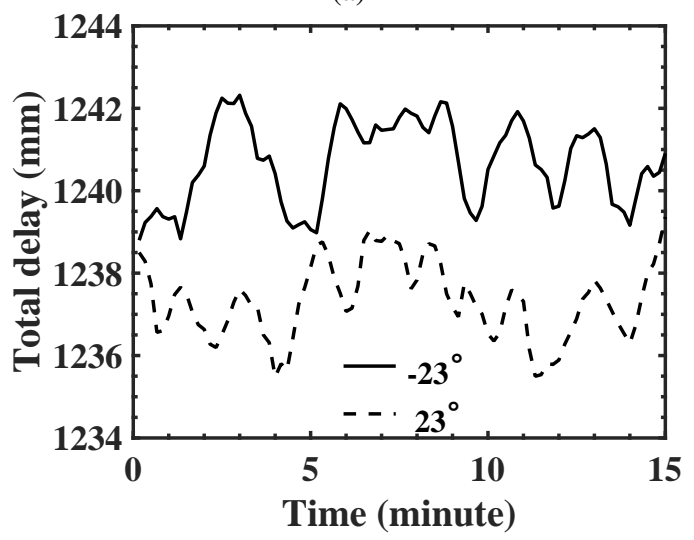

(b)

Fig. 10: Total slant delay time series for one pixel for two viewing angles $\left(-23^{\circ}\right.$ and $23^{\circ}$, angular difference $\left.46^{\circ}\right)$ for (a) the most changing pixel, and (b) the least changing pixel in the domain. Note the differences in range on the vertical axis.

weather circumstances in this simulation.

Considering the slant tropospheric interferograms, the linear trend in delay due to the incidence angle can be neglected since it is a common term between subsequent acquisitions. ${ }^{2}$ 15-minute tropospheric interferograms with heading angles of $300^{\circ}$ and $120^{\circ}$, showing the interferograms of ascending and descending geometry with an angular difference of $46^{\circ}$ are shown in Figs. 11a and b. Zoomed-in subsets are shown in Figs. 11c and d, which show quite a significant delay difference due to the different viewing geometries.

Fig. 12 visualizes the spatial variograms for each epoch, showing that the semi-variance increases as time goes by, a sill at scales of $1500 \mathrm{~m}$, and periodicity in the variance in space and time. Correlation coefficient time series can be an indicator to express temporal variations. The first time series is obtained by comparing the data per epoch with the first one, showing the effect of the cumulative total slant delay, as shown in Fig. 13a. This shows that the correlation coefficient drops to less than 0.1 in 5 minutes, which is near-decorrelation. The second correlation coefficient time series is obtained from subsequent

\footnotetext{
${ }^{2}$ Setting the heading to 120 and 300 degrees, the corresponding total slant delay time series and their corresponding single master and daisy chain interferograms are included in the supplementary data.
}

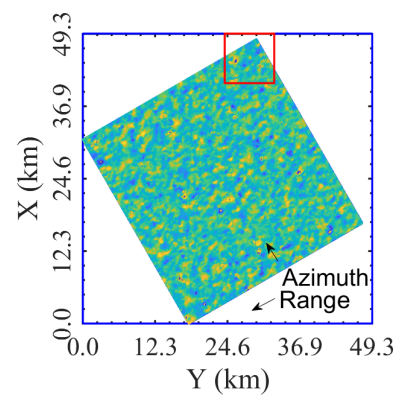

(a)

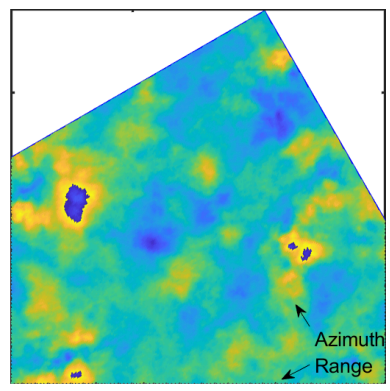

(c)

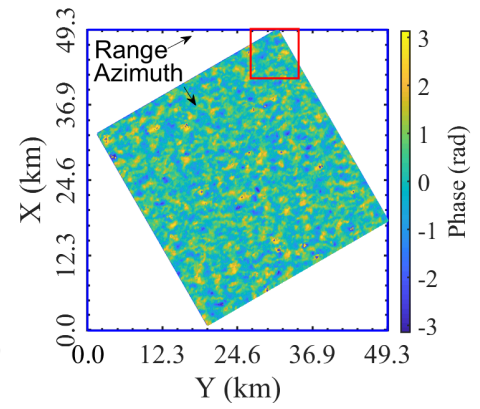

(b)

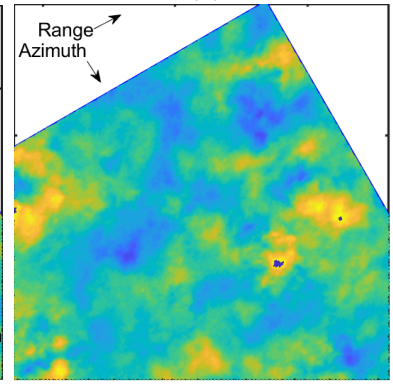

(d)
Fig. 11: 15 minute tropospheric interferogram (epoch 1-90) with different heading of (a) $300^{\circ}$ and (b) $120^{\circ}$ and their corresponding magnified views (c) and (d) (Red box in (a) and (b) denotes the test area)

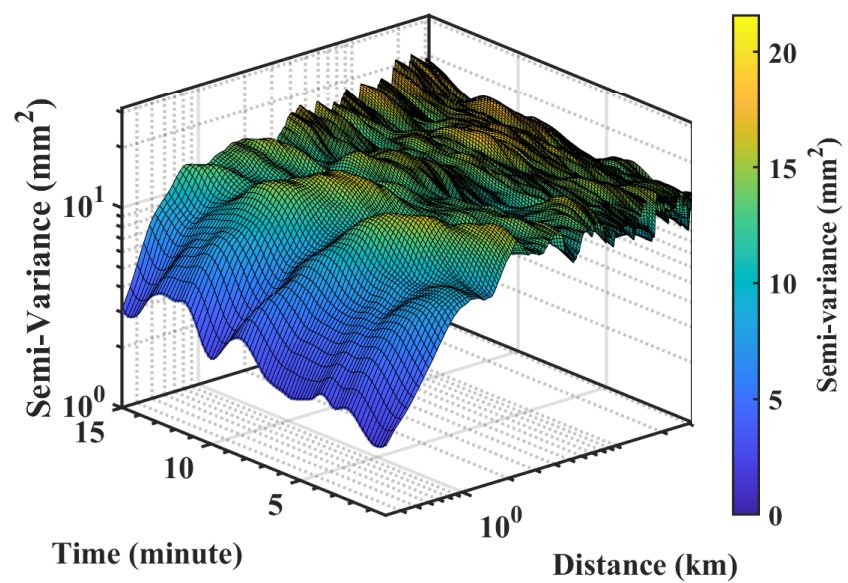

Fig. 12: Spatial variograms of the total delay for each epoch, showing a sill at a range of $\sim 1.5 \mathrm{~km}$, and temporal and spatial periodicity

(daisy-chain) acquisitions, only sensitive to fast changes in the troposphere, see Fig. 13b. These correlation coefficients are larger than 0.9 over time, indicating that temporal changes in tropospheric delay in 10 seconds can be neglected for the simulated weather conditions.

Temporal variation of the troposphere leads to fast decorrelation for the same location. The dominant variability is due to the heterogeneous spatial distribution of water vapor, of which clouds can be regarded as tracer. Assuming that this distribution is moving uniformly during the simulated 


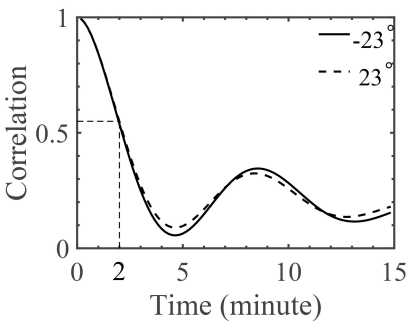

(a)

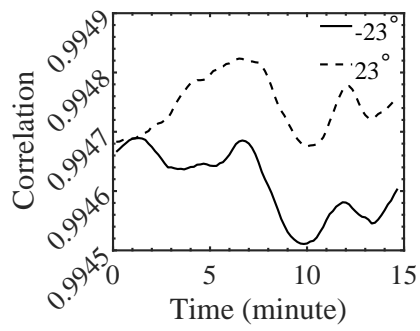

(b)
Fig. 13: Correlation coefficient time series relative to (a) the first epoch and (b) the previous epoch $(t-10 \mathrm{sec})$. Expressed for two viewing angles with an angular difference of $46^{\circ}$

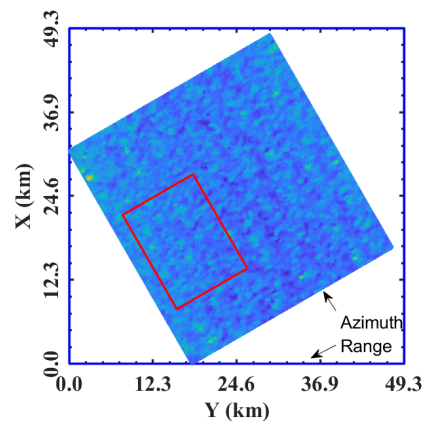

(a)

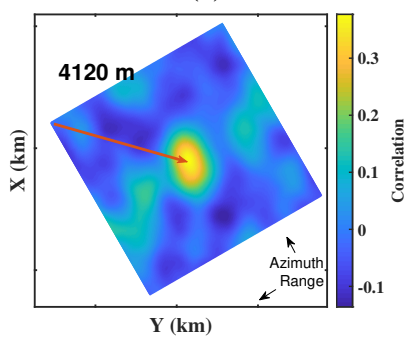

(c)

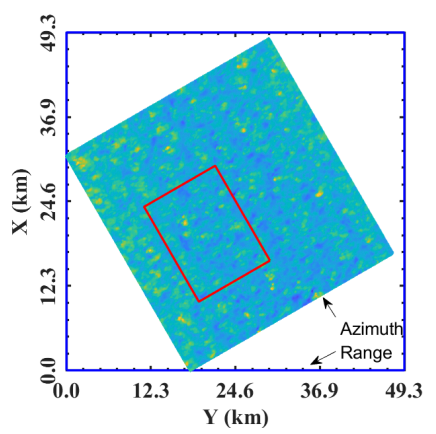

(b)

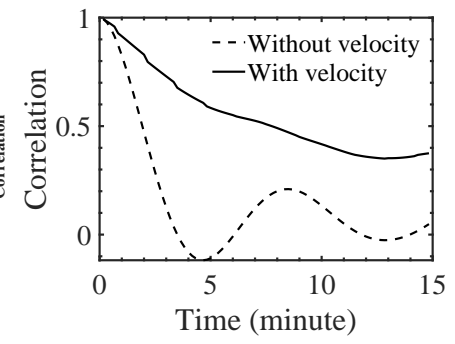

(d)
Fig. 14: Spatial correlation between total slant delay in the first and last epoch. Total slant delay in the first epoch (a) and last epoch (b) (Red box in (a) and (b) denote the test area). (c) Correlation coefficient in the search window. Red arrow indicates the the shift due to the 'frozen flow'. (d) Correlation time series in the test area.

period, using Taylor's hypothesis, [20], we expect a potential spatial correlation along the direction of motion. A test area of $16 \times 12 \mathrm{~km}$ is selected to calculate the velocity of the water vapor distribution. Setting the search window to $4 \times 4 \mathrm{~km}$, the correlation matrix between the total delay in the first and last epoch within the search window is obtained, which is shown in Fig. 14c. The location of the water vapor anomalies in different epochs is shown in Figs. 14a and b, and is identified by the largest correlation coefficient. We derive that the water vapor distribution is moving in the northeastern direction with an estimated velocity of $4.6 \mathrm{~m} / \mathrm{s}$. Using this estimated velocity, the corrected correlation coefficient time series are obtained, which are shown in Fig. 14d. Compared with the result without velocity correction, cf. Fig. 13a, the corrected one shows less loss of correlation, suggesting that the decorrelation is dominantly a result of the translation of the entire refractivity distribution as a result of the prevailing wind direction.

\section{Atmosphere Correction Using Lower Resolution Interfero- gram}

During GEOSAR radar imaging, the tropospheric delay may lead to decorrelation in case of long integration times required for high-resolution imaging. Here, we set the integration time to $100,225,450$ and 900 seconds and produce the corresponding interferograms with spatial resolutions of 250 , 120,60 and $30 \mathrm{~m}$, respectively. The total delay boxplots with different integration times are shown in Fig. 15, indicating that the variation of the total slant delay during the original integration time $(900 \mathrm{~s})$ exceeds $30 \mathrm{~mm}$. If the integration time is limited to $100 \mathrm{~s}$, the change in the total delay ranges from -10 to $10 \mathrm{~mm}$, which is smaller than one cycle. Thus, interferograms with shorter integration times will suffer less from tropospheric delay.

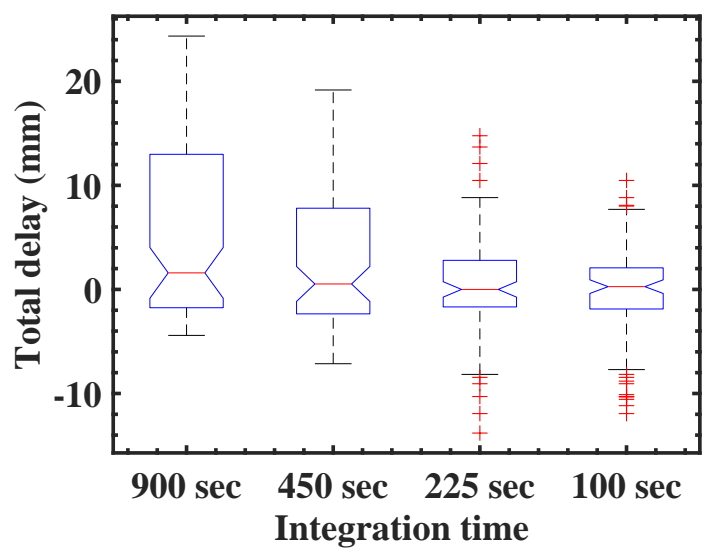

Fig. 15: Variation of the total delay for one location with different integration times.

To investigate the effect of the interferogram resolution, the temporal variations of the total slant delay in interferograms with different spatial resolutions, over the same area, are shown in Fig 16. The total slant delay in the $30 \mathrm{~m}$ interferogram ranges from 1230 to $1248 \mathrm{~mm}$ while that in the $250 \mathrm{~m}$ interferogram ranges from 1232 to $1242 \mathrm{~mm}$, showing that the dynamics of he troposphere has a smaller effect on radar imaging with lower image resolution. Figs. 18a, $\mathrm{b}$, and c show the 15 ' single master interferograms consisting of images with different integration times, showing that the spatial variation decreases for lower resolution interferograms (fast focusing). The phase-time variation of interferograms with different resolution for each epoch are shown in Fig. 17.

In GEOSAR radar imaging, high resolution requires a long integration time, suffering more from the troposphere dynamics, while it has a less influence on lower resolution images due to the shorter integration time. As a result, the refractivity distribution can be estimated in first order from a low resolution image, which is subsequently used for atmospheric correction of the high resolution image. Based on 


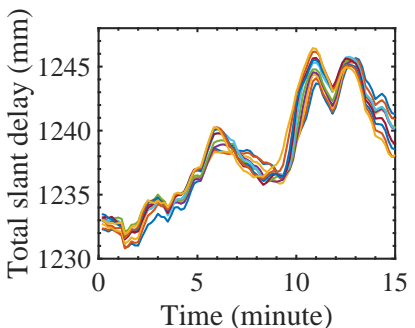

(a)

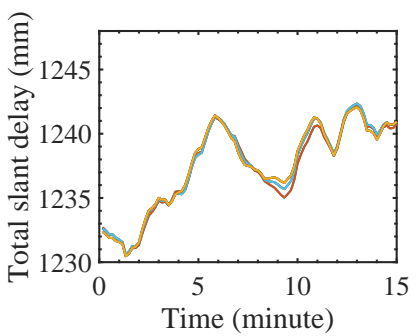

(c)

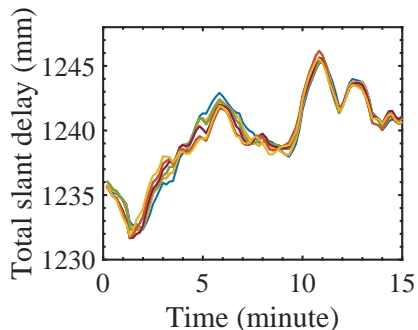

(b)

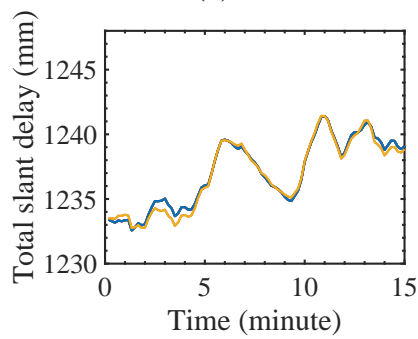

(d)
Fig. 16: Total slant delay time series of selected pixels in a common area on the ground, expressed with different colors, for interferograms with different resolution (a) $30 \mathrm{~m}, 11$ pixels, (b) $60 \mathrm{~m}, 6$ pixels, (c) $120 \mathrm{~m}, 3$ pixels, and (d) $250 \mathrm{~m}, 2$ pixels.

interferograms with the four resolutions (900, 450, 225, and $100 \mathrm{~s}$ in Figs. 11a and 18a, b, and c, respectively), there are six potential combinations for multi-scale atmospheric correction of the 15' single master interferogram. The effectiveness of the atmospheric correction can be evaluated using the phase residuals of the 15' compensated interferogram, which is shown in Fig. 19. Comparing Figs. 19a, b, and c shows that the larger the difference is between the original and the corrected interferogram resolution, the higher phase SD we get. Phase residuals of the $30 \mathrm{~m}$ interferogram corrected by the $60 \mathrm{~m}$ interferogram are less than $2 \mathrm{~mm}$, while a correction by the $250 \mathrm{~m}$ interferogram leads to phase residuals exceeding $5 \mathrm{~mm}$. Thus, the effects of the troposphere dynamics during radar imaging with long integration times can be well eliminated using the integrated refractivity estimated by interferograms with shorter integration times.

\section{Conclusions}

Based on a Large Eddy Simulation model (DALES) for a fair-weather situation with shallow cumulus convection, we provide a model of the water-vapor time-space evolution that is very useful for assessing the impact of tropospheric delay on "(GEOSAR) inteferograms". A DALES-InSAR toolbox is developed to simulate atmospheric phase screens from the parameters of DALES and to generate both vertical and slant delay interferograms with specific orbital and viewing parameters, available via GITHUB. ${ }^{3}$ The results confirm that the wet delay contributes more to the spatio-temporal variation of the tropospheric delay than the hydrostatic delay. For time intervals up to 15 minutes, the tropospheric signal can be considered as a frozen-flow signal, which can be corrected for,

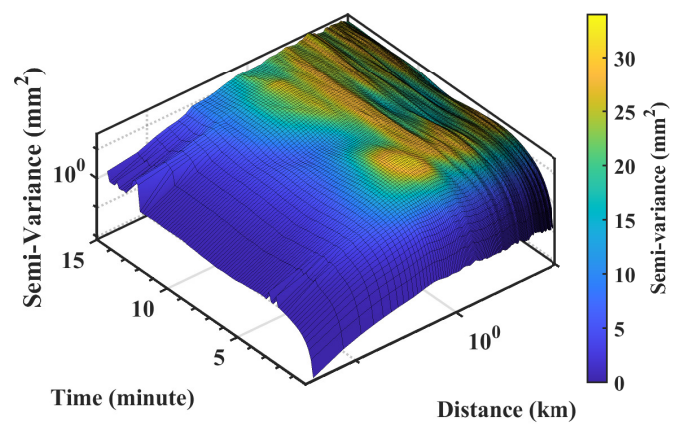

(a)

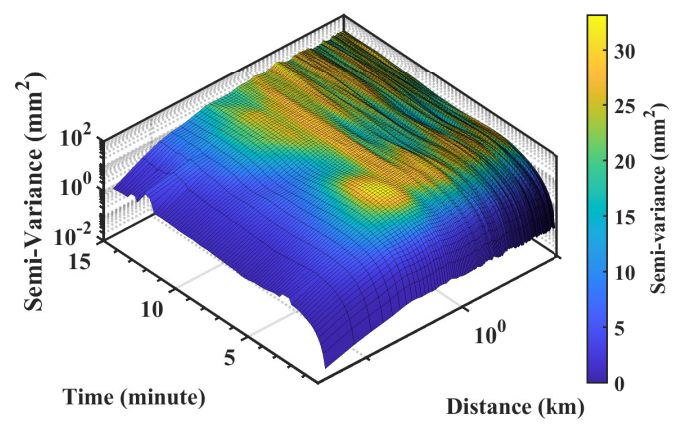

(b)

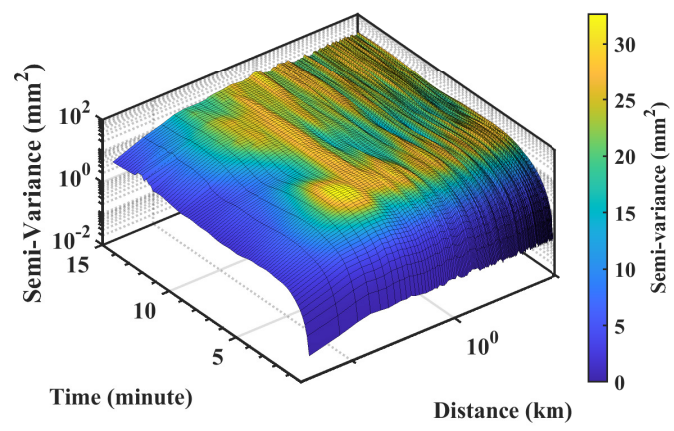

(c)

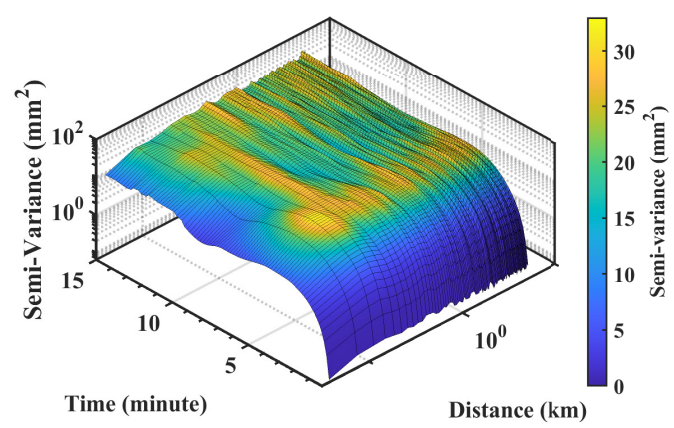

(d)

Fig. 17: Spatial variograms of the single master interferograms for each epoch, with resolutions (a) $30 \mathrm{~m} \mathrm{(b)} 60 \mathrm{~m}$ (c) $120 \mathrm{~m}$ (d) $250 \mathrm{~m}$. 


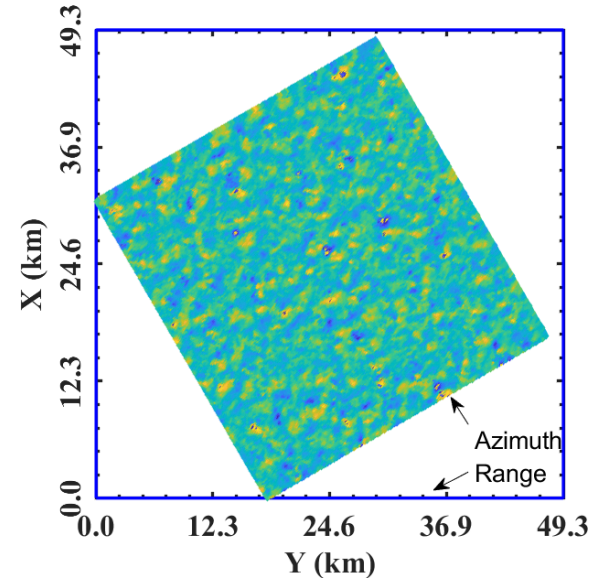

(a)

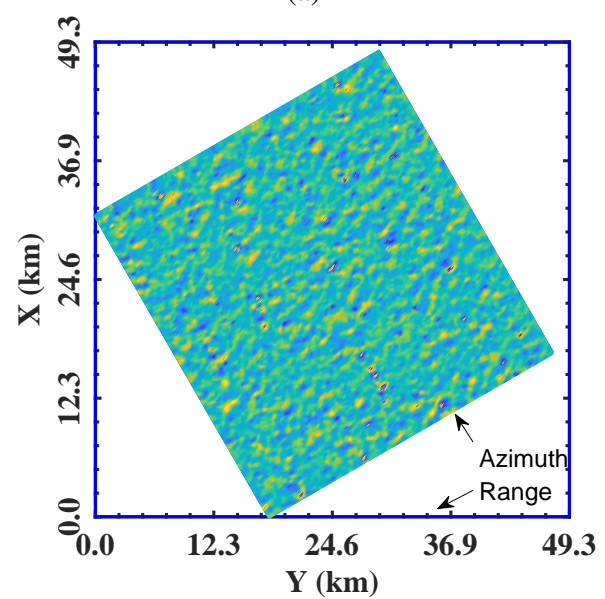

(b)



(c)

Fig. 18: 15 minute single master interferograms of slant delay with different integration time (a) $450 \mathrm{~s}$ (epoch 46-90) (b) $225 \mathrm{~s}$ (epoch 68-90) (c) $100 \mathrm{~s}$ (epoch 81-90). but for longer time intervals turbulent advection becomes the dominant part of the signal. Without a frozen-flow correction, the maximum delay variation can be more than $30 \mathrm{~mm}$ during the 15-minute acquisition, leading to total decorrelation of the radar image. During GEOSAR radar imaging, interferograms with shorter integration times, i.e. lower resolution, suffer less from tropospheric delay variability. Thus, radar imaging of GEOSAR systems should be iterative so that the tropospheric effects in the interferograms with long integration time can be compensated using the integrated refractivity obtained by the interfergrams with a shorter integration time, to decrease the decorrelation.

As a final remark, we underline that the proposed methodology and metrics have been demonstrated to only one weather situation, and should be repeated to a broader range of situations.

\section{ACKNOWLEDGMENT}

The authors would like to thank S. Hobbs from Cranfield University and A. Monti-Guarnieri from Politecnico di Milano for support and valuable suggestions. This research was funded with the Hydroterra Phase-0 Science and Requirement project by ESA. DALES is opensource software, available under the terms of the GNU GPL version 3 at https://github.com/dalesteam/dales. The DALES-InSAR codes are available at https://github.com/dogfisher/DALES_Atmosphere.

\section{REFERENCES}

[1] R. Bamler and P. Hartl, "Synthetic aperture radar interferometry," Inverse Problems, vol. 14, pp. R1-R54, 1998. [Online]. Available: http://doris.tudelft.nl/Literature/bamler98.pdf

[2] R. F. Hanssen, Radar Interferometry: Data Interpretation and Error Analysis. Dordrecht: Kluwer Academic Publishers, 2001.

[3] T. Kobayashi, Y. Morishita, and H. Yarai, "Detailed crustal deformation and fault rupture of the 2015 gorkha earthquake, nepal, revealed from scansar-based interferograms of alos-2," Earth, Planets and Space, vol. 67, no. 1, p. 201, 2015.

[4] A. Hooper, P. Segall, and H. Zebker, "Persistent scatterer interferometric synthetic aperture radar for crustal deformation analysis, with application to Volcán Alcedo, Galápagos," Journal of Geophysical Research: Solid Earth, vol. 112, no. B7, 2007.

[5] B. Zhang, R. Wang, Y. Deng, P. Ma, H. Lin, and J. Wang, "Mapping the yellow river delta land subsidence with multitemporal sar interferometry by exploiting both persistent and distributed scatterers," ISPRS Journal of Photogrammetry and Remote Sensing, vol. 148, pp. 157173, 2019.

[6] R. F. Hanssen, T. M. Weckwerth, H. A. Zebker, and R. Klees, "High-resolution water vapor mapping from interferometric radar measurements," Science, vol. 283, pp. 1295-1297, Feb.-26 1999. [Online]. Available: http://doris.tudelft.n1/Literature/hanssen99.pdf 


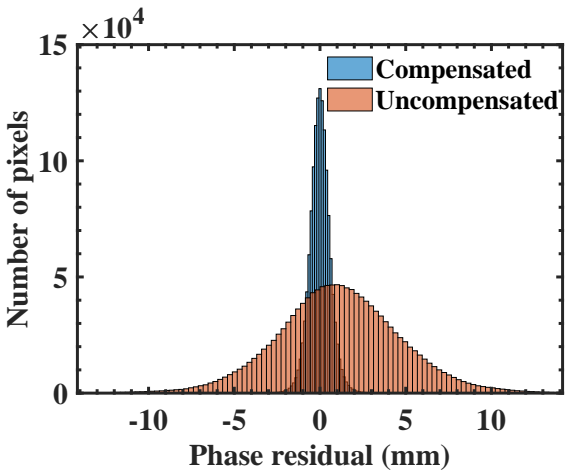

(a)

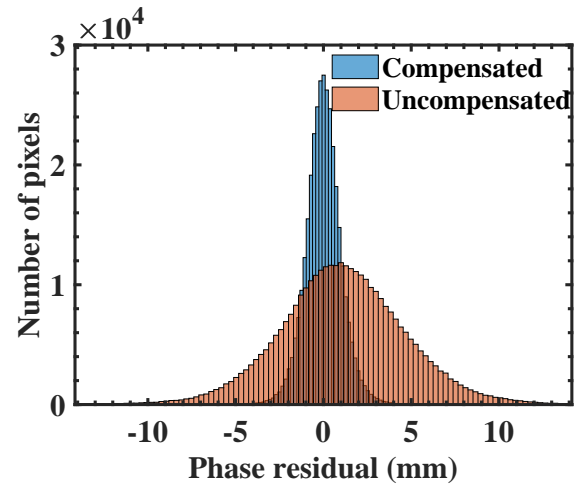

(d)

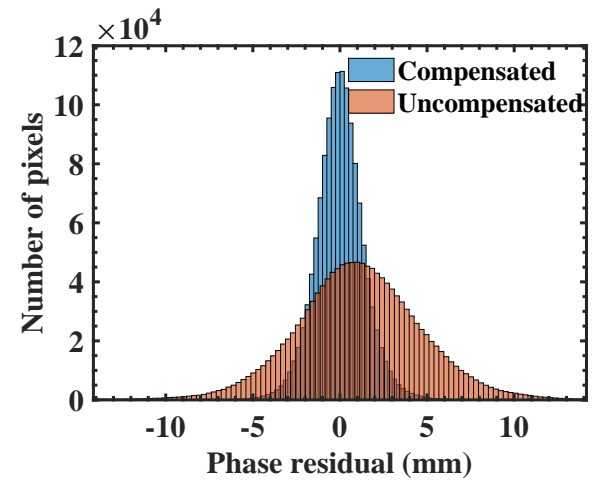

(b)

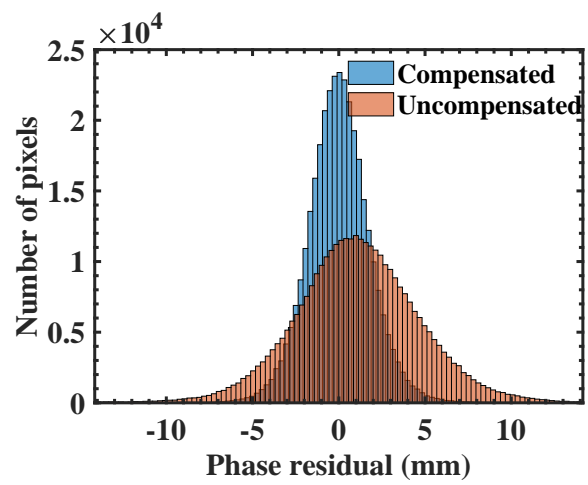

(e)

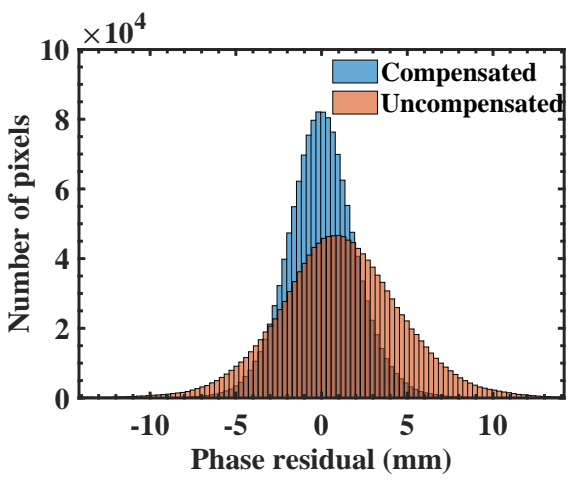

(c)

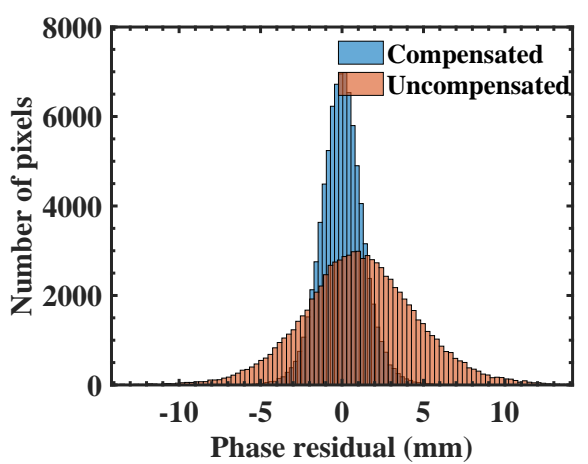

(f)

Fig. 19: Effect of integrated refractivity resolution on the phase residual of the compensated interferogram (15 minute single master interferogram). (a)-(c) are compensated $30 \mathrm{~m}$ interferograms by $60 \mathrm{~m}, 120 \mathrm{~m}$ and $250 \mathrm{~m}$ integrated refractivity. (d) and (e) are compensated $60 \mathrm{~m}$ interferograms by $120 \mathrm{~m}$ and $250 \mathrm{~m}$ integrated refractivity. (f) is compensated $120 \mathrm{~m}$ interferograms by $250 \mathrm{~m}$ integrated refractivity.

[7] P. Mateus, J. Catalão, G. Nico, and P. Benevides, "Mapping precipitable water vapor time series from sentinel-1 interferometric sar," IEEE Transactions on Geoscience and Remote Sensing, vol. 58, no. 2, pp. 1373-1379, Feb 2020.

[8] P. Mateus, R. Tomé, G. Nico, and J. Catalão, “Threedimensional variational assimilation of insar pwv using the wrfda model," IEEE Transactions on Geoscience and Remote Sensing, vol. 54, no. 12, pp. 7323-7330, Dec 2016.

[9] P. Mateus, P. M. A. Miranda, G. Nico, J. P. S. Catalao, P. Pinto, and R. Tome, "Assimilating insar maps of water vapor to improve heavy rainfall forecasts: A case study with two successive storms," Journal of Geophysical Research, vol. 123, no. 7, pp. 3341-3355, 2018.

[10] K. Tomiyasu, "Synthetic aperture radar in geosynchronous orbit," in 1978 Antennas and Propagation Society International Symposium, vol. 16, 1978, pp. 4245.

[11] R. Haagmans, J.-C. Calvet, G. Halloran, R. Hanssen, S. Hobbs, J. M. Lopez-Sanchez, F. Mattia, A. Monti Guarnieri, A. Moreira, T. Nagler et al., "Earth explorer 10 candidate mission hydroterra, esa report for assessment," 2020.

[12] M. Lagasio, A. N. Meroni, G. Boni, L. Pulvirenti,
A. Monti-Guarnieri, R. Haagmans, S. Hobbs, and A. Parodi, "Meteorological osses for new zenith total delay observations: Impact assessment for the hydroterra geosynchronous satellite on the october 2019 genoa event," Remote Sensing, vol. 12, no. 22, p. 3787, 2020.

[13] H. A. Zebker, P. A. Rosen, and S. Hensley, "Atmospheric effects in interferometric synthetic aperture radar surface deformation and topographic maps," Journal of Geophysical Research, vol. 102, no. B4, pp. 7547-7563, Apr. 1997.

[14] S. Quegan and J. Lamont, "Ionospheric and tropospheric effects on synthetic aperture radar performance," International Journal of Remote Sensing, vol. 7, no. 4, pp. 525-539, 1986.

[15] J. Saastamoinen, "Introduction to practical computation of astronomical refraction," Bulletin Geodesique, vol. 106, pp. 383-397, 1972.

[16] J. R. Elliott, J. Biggs, B. Parsons, and T. J. Wright, "InSAR slip rate determination on the altyn tagh fault, northern tibet, in the presence of topographically correlated atmospheric delays," Geophysical Research Letters, vol. 35, no. 12, 2008.

[17] D. P. S. Bekaert, A. Hooper, and T. J. Wright, "A spatially variable power law tropospheric correction technique for InSAR data," Journal of Geophysical Research: Solid 
Earth, vol. 120, no. 2, pp. 1345-1356, 2015.

[18] F. Onn and H. A. Zebker, "Correction for interferometric synthetic aperture radar atmospheric phase artifacts using time series of zenith wet delay observations from a gps network," Journal of Geophysical Research: Solid Earth, vol. 111, no. B9, 2006.

[19] Z. Li, E. J. Fielding, P. Cross, and R. Preusker, "Advanced insar atmospheric correction: Meris/modis combination and stacked water vapour models," Journal of remote sensing, vol. 30, no. 13, pp. 3343-3363, 2009.

[20] G. I. Taylor, "The spectrum of turbulence," Proceedings of the Royal Society of London. Series A-Mathematical and Physical Sciences, vol. 164, no. 919, pp. 476-490, 1938.

[21] D. Bruno, S. E. Hobbs, and G. Ottavianelli, "Geosynchronous synthetic aperture radar: Concept design, properties and possible applications," Acta Astronautica., vol. 59, no. 1, pp. $149-156,2006$.

[22] S. Hobbs, C. Mitchell, B. Forte, R. Holley, B. Snapir, and P. Whittaker, "System design for geosynchronous synthetic aperture radar missions," IEEE Transactions on Geoscience and Remote Sensing, vol. 52, no. 12, pp. 7750-7763, 2014.

[23] F. Zhang, G. Li, W. Li, and W. Hu, "Multiband microwave imaging analysis of ionosphere and troposphere refraction for spaceborne sar," International Journal of Antennas and Propagation, vol. 2014, pp. 1-9, 2014.

[24] S. Jinping, B. Yuekai, W. Yanping, and H. Wen, "High resolution sar performance limitation by the change of tropospheric refractivity," vol. 2, pp. 1448-1451, 2011.

[25] X. Dong, J. Hu, C. Hu, T. Long, Y. Li, and Y. Tian, "Modeling and quantitative analysis of tropospheric impact on inclined geosynchronous sar imaging," Remote Sensing, vol. 11, p. 803, 042019.

[26] D. Bruno and S. E. Hobbs, "Radar imaging from geosynchronous orbit: Temporal decorrelation aspects," IEEE Transactions on Geoscience and Remote Sensing, vol. 48, no. 7, pp. 2924-2929, 2010.

[27] M. Zhu, G. Wadge, R. Holley, I. James, P. Clark, C. Wang, and M. Woodage, "High-resolution forecast models of water vapor over mountains: Comparison with meris and meteosat data," IEEE Geoscience and Remote Sensing Letters, vol. 4, pp. 401 - 405, 082007.

[28] A. M. Guarnieri, S. Tebaldini, F. Rocca, and A. Broquetas, "Gemini: Geosynchronous sar for earth monitoring by interferometry and imaging," in 2012 IEEE International Geoscience and Remote Sensing Symposium, 2012, pp. 210-213.

[29] A. Monti Guarnieri, A. Leanza, A. Recchia, S. Tebaldini, and G. Venuti, "Atmospheric phase screen in geo-sar: Estimation and compensation," IEEE Transactions on Geoscience and Remote Sensing, vol. 56, no. 3, pp. 1668-1679, March 2018.

[30] J. Wallace and P. Hobbs, Atmosphere science - an introductory survey. Elsevier, 1997.

[31] R. Hanssen and R. Klees, "Refractivity maps obtained by radar interferometry," UNAVCO Annual Meeting, 11-13 May, Boulder CO, 1998.
[32] T. Heus, C. C. van Heerwaarden, H. J. J. Jonker, A. Pier Siebesma, S. Axelsen, K. van den Dries, O. Geoffroy, A. F. Moene, D. Pino, S. R. de Roode, and J. Vilà-Guerau de Arellano, "Formulation of the dutch atmospheric large-eddy simulation (dales) and overview of its applications," Geoscientific Model Development, vol. 3, no. 2, pp. 415-444, 2010. [Online]. Available: https://gmd.copernicus.org/articles/3/415/2010/

[33] A. K. Betts, "Non-precipitating cumulus convection and its parameterization," Quart.j.roy.meteor.soc, vol. 99, no. 419, pp. 178-196, 1973.

[34] L. Urquhart, F. Geremia-Nievinski, and M. Santos, "Raytraced slant factors for mitigating the tropospheric delay at the observation level," Journal of Geodesy, vol. 86, pp. 149-160, 022011.

[35] B. Parkinson, P. Axelrad, and P. Enge, Global Positioning System Theory and Applications. AAIA: Washington, DC, USA, 1996.

[36] F. Alshawaf, S. Hinz, M. Mayer, and F. J. Meyer, "Constructing accurate maps of atmospheric water vapor by combining interferometric synthetic aperture radar and gnss observations," Journal of Geophysical Research, vol. 120, no. 4, pp. 1391-1403, 2015.

[37] E. Pichelli, R. Ferretti, D. Cimini, G. Panegrossi, D. Perissin, N. Pierdicca, F. Rocca, and B. Rommen, "Insar water vapor data assimilation into mesoscale model mm5: Technique and pilot study," IEEE Journal of Selected Topics in Applied Earth Observations and Remote Sensing, vol. 8, no. 8, pp. 3859-3875, Aug 2015.

[38] A. R. Brown, R. T. Cederwall, A. Chlond, P. G. Duynkerke, J. Golaz, M. Khairoutdinov, D. C. Lewellen, A. P. Lock, M. K. Macvean, C. Moeng et al., "Largeeddy simulation of the diurnal cycle of shallow cumulus convection over land," Quarterly Journal of the Royal Meteorological Society, vol. 128, no. 582, pp. 10751093, 2002. 